\title{
Mixing the Light Spin with Plasmon Orbit by Nonlinear Light-Matter Interaction in Gold
}

\author{
G. Spektor, ${ }^{1, *}$ D. Kilbane, ${ }^{2,3,4}$ A. K. Mahro, ${ }^{2}$ M. Hartelt, ${ }^{2}$ E. Prinz,,${ }^{2,5}$ M. Aeschlimann, ${ }^{2}$ and M. Orenstein ${ }^{1}$ \\ ${ }^{1}$ Department of Electrical Engineering, Technion-Israel Institute of Technology, 32000 Haifa, Israel \\ ${ }^{2}$ Department of Physics and Research Center OPTIMAS, University of Kaiserslautern, \\ Erwin Schroedinger Strasse 46, 67663 Kaiserslautern, Germany \\ ${ }^{3}$ School of Physics, University College Dublin, Belfield, Dublin 4, Ireland \\ ${ }^{4}$ Telecommunications Software \& Systems Group, Waterford Institute of Technology, Waterford, Ireland \\ ${ }^{5}$ Graduate School Materials Science in Mainz, Staudingerweg 9, 55128 Mainz, Germany
}

(Received 18 November 2018; published 15 May 2019)

\begin{abstract}
Transformation of light carrying spin angular momentum (SAM) to optical field vortices carrying orbital angular momentum (OAM) has been of wide interest in recent years. The interactions between two optical fields, each carrying one of those degrees of freedom, and furthermore, the transfer of the resulting angular momentum product to matter are seldom discussed. Here, we measure the interaction between 3D light carrying axial SAM and 2D plasmon-polariton vortices carrying high-order transverse OAM. The interaction is mediated by two-photon absorption within a gold surface, imprinting the resulting angular-momentum mixing into matter by excitation of electrons that are photo-emitted into vacuum. Interestingly, the spatial distribution of the emitted electrons carries the signature of a subtraction of the spin from the orbit angular momenta. We show experimentally and theoretically that the absorptive nature of this interaction leads to both single and double photon-plasmon angular momentum mixing processes by one- and two- photon interactions. Our results demonstrate high order angular momenta light-matter interactions, provide a glimpse into specific electronic excitation routes, and may be applied in future electronic sources and coherent control.
\end{abstract}

DOI: 10.1103/PhysRevX.9.021031

Subject Areas: Photonics, Plasmonics,

Quantum Physics

\section{INTRODUCTION}

Optical fields can carry what is referred to as spin angular momentum (SAM) in the form of circularly polarized light and orbital angular momentum (OAM) in the form of optical vortices [1-3]. The transformation between these two degrees of freedom has led to the discovery of new phenomena [4] and opened a route to many promising applications [5]. In particular, by applying 2D chiral boundary conditions, 3D circularly polarized light carrying SAM can be converted into 2D surface plasmon polariton (SPP) vortices, exhibiting surfaceconfined orbital angular momentum [6-10]. SPPs are 2D surface waves confined to metal-dielectric interfaces and can exhibit a variety of angular-momentum-related properties [11-15]. The plasmonic vortices can be used as optical tweezers for particle manipulations [16-18], and they have solid promise in the delivery of high-order

\footnotetext{
*spektorg@campus.technion.ac.il
}

Published by the American Physical Society under the terms of the Creative Commons Attribution 4.0 International license. Further distribution of this work must maintain attribution to the author(s) and the published article's title, journal citation, and DOI. angular-momentum quanta to electronic degrees of freedom $[19,20]$. The typical spin-to-orbit conversion processes are based on local and linear interactions and require chiral structures such as edges and shaped slits such as spirals [21-24], or particles [25]. Nonlinearities in the context of angular momenta include OAM conservation by harmonic generation [26-30] and stimulated downconversion [31], as well as generation of harmonic far-field vortices as refractions from nonlinear fork-shaped photonic crystals $[32,33]$. Distributed nano-antennas were shown to produce local spin-orbit conversions [34,35] and form high-harmonic OAM beams from circularly polarized illumination [34,36,37]. Such antennas typically act as local point sources for harmonic generation, which then linearly interfere to produce far-field vortices. However, the mixing of angular momenta between two existing fields, each carrying a different form of angular momentum, has not been previously discussed.

Here, we present experimentally the realization of direct optical spin-orbit mixing. We implement a mixing configuration based on the interaction between 3D light and 2D plasmons, carrying, respectively, spin and orbital-angular momenta [Fig. 1(a)]. The mixing outcome is transferred to matter via a two-photon absorption process, yielding electrons that are emanated from a gold surface, without 


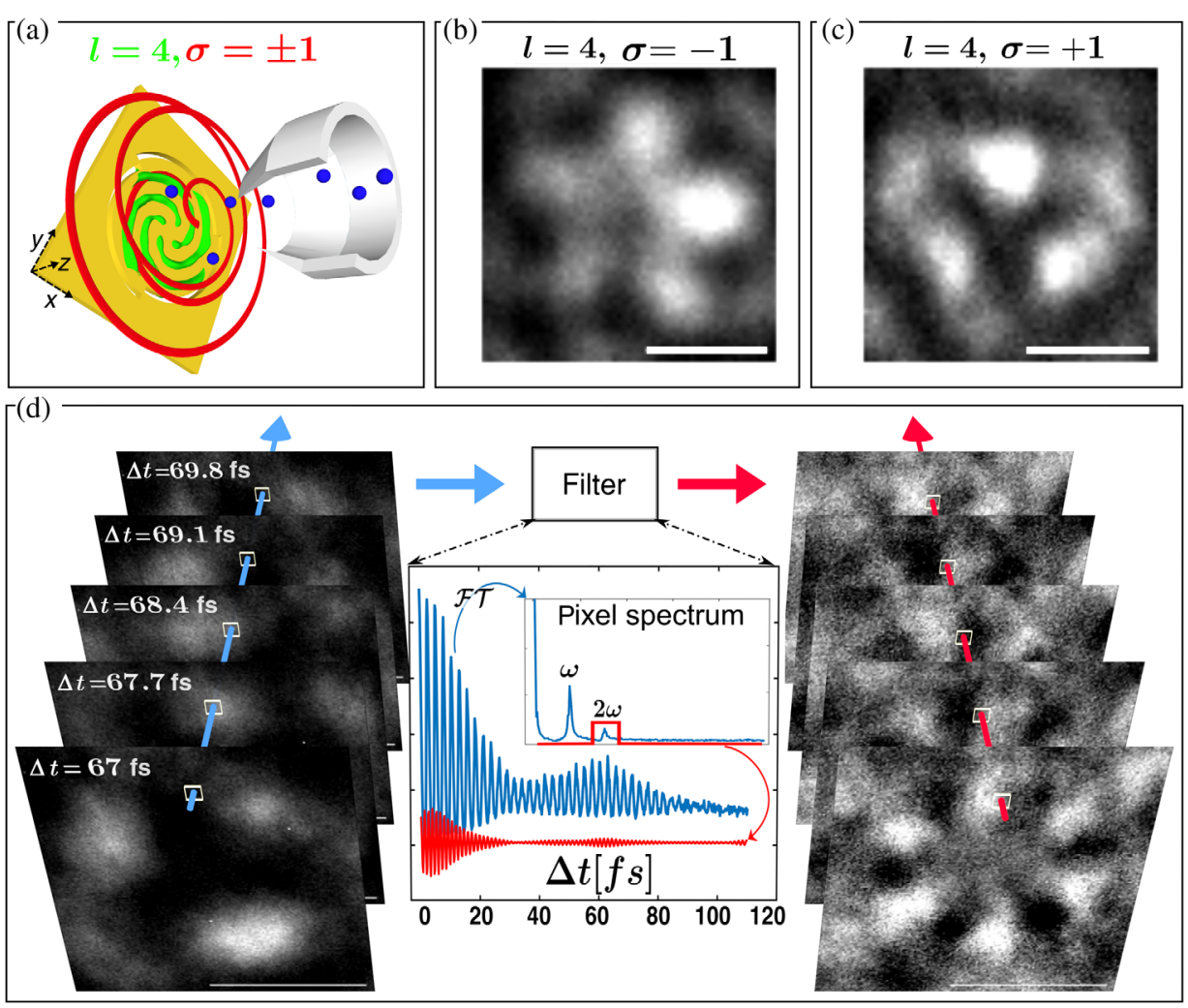

FIG. 1. Experimental concept and results schematic. (a) Plasmonic vortex of order $l=4$ (green) revolving on a gold surface and interacting with right or left circularly polarized light pulses $(\sigma=+1$ or -1$)$ (red helix). The blue circles represent the photoemitted electrons resulting from two-photon absorption. (b,c) Raw experimental data of delay-time snapshots of electron emission patterns exhibiting an angular distribution of order 5 (b) and 3 (c), indicating the subtraction of the plasmon and light angular momenta. (d) Isolating a double light-plasmon mixing process. The raw data of electron distribution images at varying plasmon-light time delay (left sequence, light-blue guiding arrow) are pixelwise filtered at twice the optical frequency $\omega$ (right sequence, red guiding arrow). This case unveils an OAM pattern with twice the lobes of the dominant data. The central graph demonstrates the filtering procedure, taking the raw pixel values for all measured delay values (light blue) and isolating the $2 \omega$ oscillating term (red). The scale bar on all data images is 1 micron.

any mediating structures. The electrons are collected throughout the entire 2D surface area, providing imaging of the interaction at a high spatial resolution. The multilobed angular distribution of the photoemission pattern corresponds to a specific angular-momentum combination of the $3 \mathrm{D}$ and $2 \mathrm{D}$ interacting fields and indicates the coupling of the light SAM and plasmonic OAM. Interestingly, the observed spatial electron distributions correspond to the subtraction of the SAM of the light field from the OAM of the plasmonic vortex [Figs. 1(b) and 1(c)] rather than their addition. We show that such a subtractive mixing effect fundamentally stems from the absorptive nature of the light-matter interaction. Finally, we unveil a second-order mixing process resulting in photoemission due to two plasmon-photon mixing events. This process doubles the azimuthal order of the spatial distribution of the photoemitted electrons, corresponding to high-order angular-momentum mixing [Fig. 1(d)].

Our results thus demonstrate light-matter interactions that adhere to orbital angular-momentum selection rules, where the local electron photoemission rates are controlled by controlling the angular momentum of any of the interacting fields. These mechanisms could lead to various applications and may have implications on Raman spectroscopy, advanced light-matter interactions, electronic sources, and coherent control.

The plasmonic vortex, owing to its $2 \mathrm{D}$ confinement and transverse magnetic nature [38,39], is not a simple scalar phase singularity but rather a complex vector field having an out-of-plane component $E_{z}$ and in-plane radial and azimuthal components $E_{r}$ and $E_{\theta}$, respectively (Fig. 2). In addition, the complete temporal evolution of the plasmonic vortex pulse is rather involved. However, once the $l$ th order vortex is formed, its fully evolved state, which we denote as the revolution stage [10], can be well approximated by [7] $E_{z} \approx B J_{l}\left(k_{\mathrm{SPP}} r\right) \times \exp (i l \theta), \quad E_{r} \approx D J_{l}^{\prime}\left(k_{\mathrm{SPP}} r\right) \times \exp (i l \theta)$, and $E_{\theta} \approx F J_{l}\left(k_{\mathrm{SPP}} r\right) \times \exp (i l \theta)$. Here, $J_{l}$ is the Bessel function of order $l$ and $J_{l}^{\prime}$ is its first derivative; $B, D$, and $F$ are complex proportionality factors that are functions of the angular light frequency $\omega$, phase, loss, and coupling 

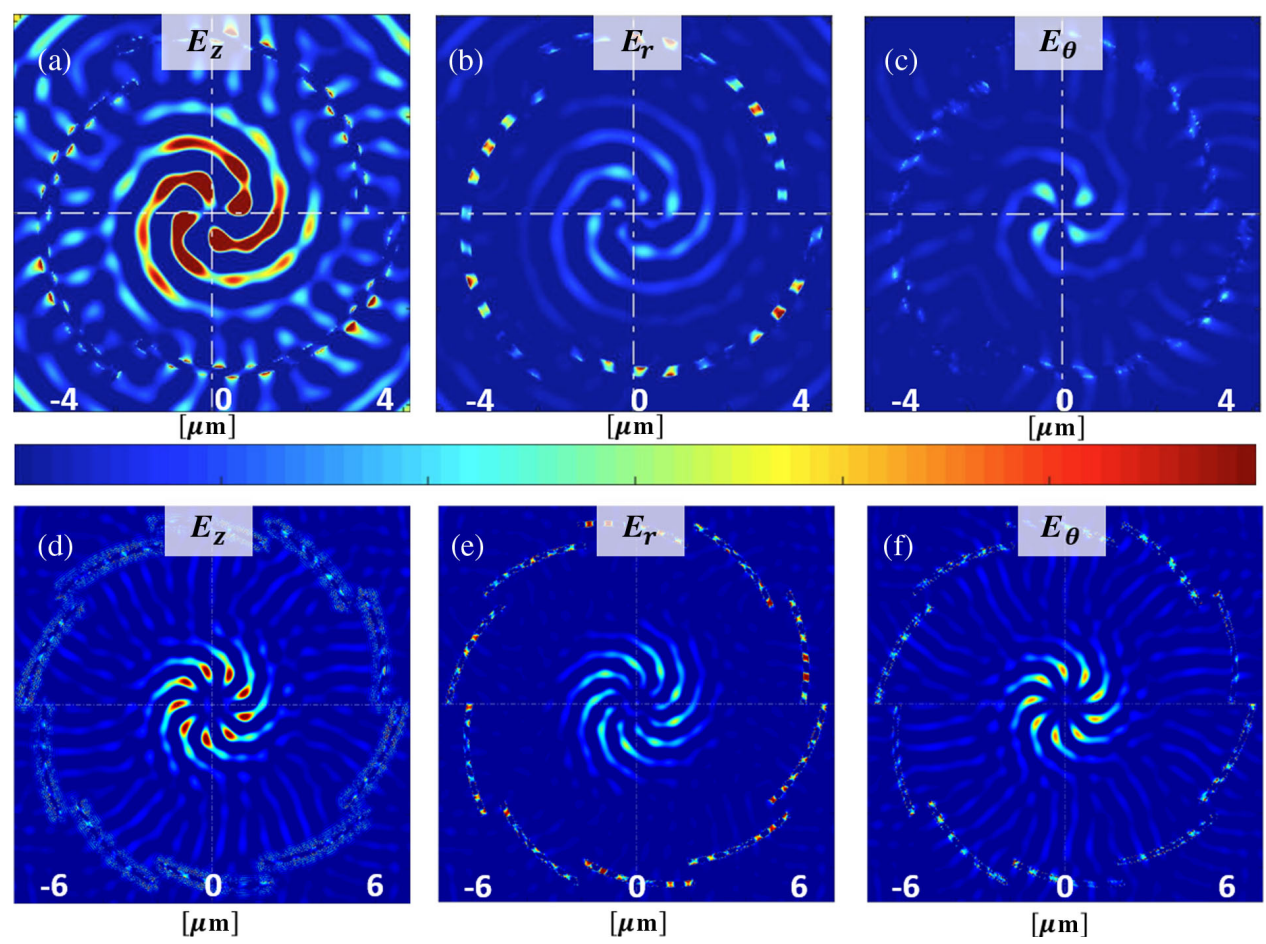

FIG. 2. Finite-difference time-domain simulation of the plasmonic vortex field. Time snapshots of the spatial distributions of vortex field components. The simulations are done within plasmonic vortex generators [7] (see SM [40]) of order $m=3$ and $m=10$ yielding $l=4[(\mathrm{a})-(\mathrm{c})]$ and $l=9[(\mathrm{~d})$-(f)] vortices, respectively. Each field component clearly shows 4 and 9 spiraling wave fronts and lobes. The fields are simulated outside the gold layer and are normalized between -1 (blue) and 1 (red) of the out-of-plane field.

coefficients; $r, \theta$, and $z$ are the cylindrical coordinates; $k_{\mathrm{SPP}}$ is the plasmonic wave number; and $i=\sqrt{-1}$ (see Supplemental Material (SM) [40] for full expressions). The azimuthal phase dependence $\exp (i l \theta)$ defines the amount of total orbital angular momentum carried by the vortex and is common to all the field components (Fig. 2). Because of the issue of the nonseparability of photonic angularmomenta terms, we refer to the angular momentum of the $l$ th order plasmon vortex as the total angular momentum, which is predominantly orbital. In the time domain, the vortex displays azimuthal rotation $\cos (\omega t-l \theta)$, exhibiting $l$ wave fronts, or lobes, that revolve around the vortex center with angular velocity of $2 \pi / l$ radians per optical cycle. The number of lobes provides a direct indication of the angular momentum $l$ carried by the vortex.

\section{RESULTS AND DISCUSSION}

Our experiment is performed by prelaunching a plasmonic vortex of order $l$ on a gold layer (plasmonic vortex launching [7] is described in the Appendix A, and plasmon excitation efficiency in Appendix B). The angular-momentum mixing starts when we subsequently illuminate the same metal surface with a light pulse of about 23 fs centered at a 800-nm wavelength, having a circular polarization of choice after an interferometrically controlled [41] delay time $\Delta t$ from the launching of the plasmonic vortex. The light pulse impinges the metal surface at a normal incidence along the $z$ axis; having a diameter of about $100 \mu \mathrm{m}$ on the sample, it spatially overlaps the whole area where the 2D plasmonic vortex revolves [Fig. 1(a)]. The light-pulse field that penetrates the metal, combined with the plasmonic vortex field, is embedded into an excited electron population by a twophoton absorption process. The generated excited electron population has energy exceeding the work function of the gold-vacuum interface (with the assistance of a subatomic layer of $\mathrm{Cs}$ ), resulting in electron emission to vacuum and their subsequent collection and imaging via electron optics in a photoemission electron microscope (PEEM) setup [Fig. 1(a)]. This configuration provides imaging of the emitted electron distribution with about 30-nm spatial resolution and 100-attosecond time-delay steps [10,42-46].

We are interested in the light SAM mixing with OAM of the plasmonic vortex; thus, we select the delay times $\Delta t$ to be within the time range for which the plasmonic vortex is already fully evolved and exhibits only azimuthal rotation with no radial propagation [10] (movies in the SM [40] show such mixing at all stages). Raw signals recorded by the PEEM are shown in Fig. 3 for two vortices, each interacting with both circular light polarizations. The azimuthal (as well as the radial) pattern of the emitted electron distribution is different from that of each of the field components (Fig. 2) of the plasmonic vortex by the number of lobes and the related spiraling wavefront threads. The output electron flux resulting from a 

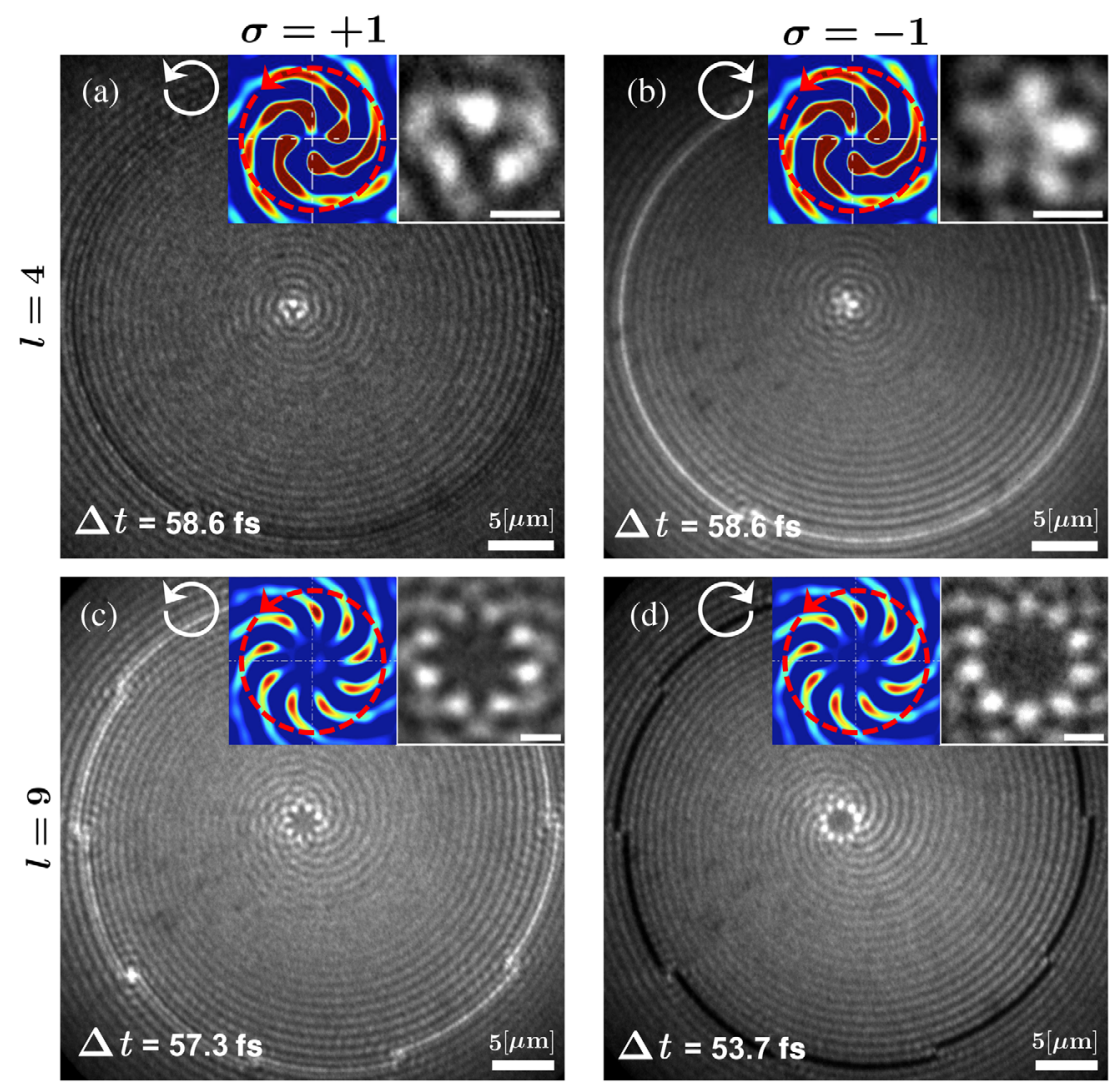

FIG. 3. Experimental raw data of spin orbit mixing of light with plasmonic vortices. The color insets are the FDTD-calculated out-ofplane field components of the interacting plasmonic vortex $l=4(\mathrm{a}, \mathrm{b})$ and $l=9(\mathrm{c}, \mathrm{d})$ for reference. The red dashed arrows represent the counter-clockwise (positive) rotation of the plasmonic vortex. The white circular arrows indicate the handedness of the polarization of the 3D light pulse: $\sigma=+1(\mathrm{a}, \mathrm{c})$ and $\sigma=-1(\mathrm{~b}, \mathrm{~d})$. The grayscale insets are zoom-ins of the center, and they show excited electron distributions having multilobes of azimuthal order 3 (a), 5 (b), 8 (c), and 10 (d). All of them indicate patterns related to $l_{\mathrm{el}}=l-\sigma$. The inset scale bar is 1 micron. All patterns revolve as a function of the light-plasmon delay time $\Delta t$ around the center of the vortex in a counter-clockwise direction (see full movies in the SM [40]).

plasmonic vortex of order $l=4$ interacting with lightfield spin of +1 and -1 exhibits azimuthally multilobed distributions of order $l_{\mathrm{el}}=3$ and $l_{\mathrm{el}}=5$, respectively [Figs. 3(a) and 3(b)]. Similarly, a plasmonic vortex of order $l=9$, when mixed with a circularly polarized light pulse, generates multilobed photo-electron distributions of order $l_{\mathrm{el}}=8$ and $l_{\mathrm{el}}=10$ for the respective light spin [Figs. 3(c) and 3(d)]. (For additional results, see SM [40].)

The resulting order of the photoemission angular pattern is thus $l_{\mathrm{el}}=l-\sigma$. Interestingly, the spin angular momentum of the light field is subtracted, rather than added, from the OAM of the plasmonic vortex when imprinted on the electronic transitions. Finally, the mixing occurs at each point of the gold surface without any mediating structures.

This mixing phenomenon can be understood from the fundamental description of the photoionization as a photoabsorption process. We start from the basic equation of motion of the system density matrix in the interaction picture, $\{[\partial \hat{\rho}(t)] / \partial t\}=[1 /(i \hbar)]\left[\hat{H}_{I}(t), \hat{\rho}(t)\right]$, where $\hat{H}_{I}$ is the light-matter-interaction Hamiltonian $\hat{H}_{I}(t) \propto \hat{p} \cdot \hat{A}$, with the momentum operator $\hat{p}$ and the vector potential operator $\hat{A}$. The probability for a one-photon ionization within a time $T$ is formally obtained by two iterative integrations yielding the nonlinear two-time process:

$$
\begin{aligned}
& P\left(t_{0}+T\right) \\
& =\frac{1}{h^{2}} \int_{t_{0}}^{t_{0}+T} d t_{1} \int_{t_{0}}^{t_{1}} d t_{2}\left\langle\Psi_{F}\left|\hat{H}_{I}\left(t_{1}\right) \hat{\rho}\left(t_{0}\right) \hat{H}_{I}\left(t_{2}\right)\right| \Psi_{F}\right\rangle+c c,
\end{aligned}
$$

where $\Psi_{F}$ is the final wave-function. Assuming a sufficiently long interaction time $\mathrm{T}$ (the pulse duration is greater than 10 optical cycles in our case) leaves only the energyconserving absorption terms, which results in the photoionization probability,

$$
\begin{aligned}
N_{1 p} & \propto T\left[\vec{\mu}_{12}^{-} \cdot \vec{E}^{+} \vec{E}^{-} \cdot \vec{\mu}_{12}^{+}+c c\right] \\
& \propto \mu_{12} \vec{e}^{-} \cdot \vec{E}^{+} \vec{E}^{-} \cdot \vec{e}^{+} \mu_{12}=\mu_{12}^{2}\left(\vec{E}^{+} \cdot \vec{E}^{*}+c c\right),
\end{aligned}
$$

where $\vec{E}^{+/-}$are the positive/negative frequency components of the total electric field, and $\vec{\mu}_{12}$ is the dipole 
transition matrix element with amplitude $\mu_{12}$. We assume here that in each point on the metal surface, the induced material dipole is aligned by the local field, and therefore, $\vec{\mu}_{12}^{-} \cdot \vec{E}^{+}=\mu_{12} \vec{e}^{-} \cdot \vec{E}^{+}$, where $\vec{e}^{+/-}$are the respective unit vectors of the electric field. The photoionization yield is thus determined by the mixing between opposite-frequency components of the induced material dipole and field. This scalar product is the core of the observed, subtractive, angular-momentum mixing process.

The total optical field inside the metal is given by $\vec{E}(r, \Delta t)=\vec{E}_{\text {light }}(r)+\vec{E}_{\mathrm{SPP}}(r, \Delta t)$, where $\Delta t$ is the delay time between the light pulse and the launch of the plasmonic vortex. Using the field of the plasmonic vortex and the circularly polarized light-field phasor in cylindrical coordinates, $\vec{E}_{\text {light }, \sigma}=E_{\text {light }}[\hat{r}+i \sigma \hat{\theta}] \exp (i \sigma \theta) / \sqrt{2}$, with $\sigma= \pm 1$ the spin of the field and $E_{\text {light }}$ the amplitude of the field within the metal, we obtain the mixing terms, terms that include scalar multiplication between light and plasmon fields, of a one-photon photoemission signal,

$$
\begin{aligned}
& N_{1 p, \text { mix }}(r, \theta, \Delta t) \\
& \quad \propto \vec{E}_{\mathrm{SPP}}^{-} \cdot \vec{E}_{\text {light }}^{+}+\vec{E}_{\text {light }}^{-} \cdot \vec{E}_{\mathrm{SPP}}^{+} \\
& \quad=E_{\mathrm{SPP}} E_{\text {light }} \cdot J_{l-\sigma}\left(k_{\mathrm{SPP}} r\right) \cos [(l-\sigma) \theta-\omega \Delta t],
\end{aligned}
$$

where $E_{\mathrm{SPP}}$ is the plasmonic field amplitude coefficient. The scalar product of the fields clearly mixes between the plasmonic orbital and light spin angular momenta, resulting in an electron-emission-rate distribution proportional to the projection of the light vector field onto the SPP vortex field. It is important to note that because of the in-plane nature of the light field, only the in-plane plasmonic components participate in the mixing process [47] (see Appendix C). The resulting spatial distribution of the emitted electrons has $l-\sigma$ azimuthal lobes, indicating the subtraction of the spin of light from the OAM of the plasmonic vortex. Thus, the subtractive spin-orbit mixing is a fundamental result of a one-photon energy- and angular-momentum conservation in the absorption process.

As an intuitively reduced interpretation, the interaction is initiated due to excitation of the electrons by one field (e.g., the light-spin carrying SAM) that generates an electronic dipole-moment rotating according to the light polarization handedness. The second field (e.g., the rotating plasmon vortex) then interacts with the rotating dipole. Thus, the vortex is essentially sampled by the nonparametric absorption process within the rotating frame of reference of the material dipole resulting in the subtraction, rather than addition, of the relative angular momentum.

Since in our experiments we employ a two-photon photoemission process, we apply the iterative integration up to fourth order, resulting in a photo-induced electronic yield distribution,

$$
\begin{aligned}
N_{2 p, \text { mix }}(r, \Delta t) \propto\left|\vec{E}^{-}(\vec{r}, \Delta t) \cdot \vec{E}^{+}(\vec{r}, \Delta t)\right|^{2}= & -\sigma\left(E_{\mathrm{SPP}}^{2}+E_{\text {light }}^{2}\right)\left(\frac{\kappa E_{\mathrm{SPP}} E_{\text {light }}}{k_{\mathrm{SPP}}}\right) J_{l-\sigma}\left(k_{\mathrm{SPP}} r\right) \cos [(l-\sigma) \theta-\omega \Delta t] \\
& -\left(\frac{\kappa E_{\mathrm{SPP}} E_{\text {light }}}{k_{\mathrm{SPP}}}\right)^{2} J_{l-\sigma}^{2}\left(k_{\mathrm{SPP}} r\right) \cos [2(l-\sigma) \theta-2 \omega \Delta t] \\
& +\left(\frac{\kappa E_{\mathrm{SPP}} E_{\text {light }}}{k_{\mathrm{SPP}}}\right)^{2} J_{l-\sigma}^{2}\left(k_{\mathrm{SPP}} r\right)
\end{aligned}
$$

where $\kappa$ is the transverse wave vector of the plasmon and only the light-plasmon mixing terms are presented. The two-photon absorption-process yield is thus comprised of three types of angular-momentum mixing interactions: The first [first term of Eq. (4)] consists of a photon-plasmon mixing event in one absorption step with absorption of either the pure plasmon or light photon in the second absorption step. The second mixing type [second term of Eq. (4)] is a double mixing process, where both absorption steps contribute to the mixing of light and plasmonic fields. Finally, the third mixing type is also a double mixing term that is accomplished by another path of light-matter excitation as described below and yields a time-delayindependent term, making it difficult to resolve experimentally from the background of the nonmixing terms [Fig. 5(j)]. The single mixing term is the one predominantly governing the raw signal for our experimental conditions (Fig. 3) and is similar to the one expected for a one-photon photoemission process [Eq. (3)]. The spin of the light pulse and orbital angular momentum of the plasmonic vortex are mixed to produce a pattern with azimuthal order related to the subtraction of the angular momenta $l_{\mathrm{el}}=l-\sigma$. The second, double mixing term results in doubling the angularmomentum values generating the electronic distribution with azimuthal order $l_{\mathrm{el}}=2(l-\sigma)$.

To experimentally resolve these two different mixing terms, we utilize the fact that the electron emission yield [Eq. (4)] has a temporal periodic dependence on the delay time between the plasmon and light-pulse launching events with angular frequencies $\omega$ and $2 \omega$ for the single and double mixing, respectively. We record the photoelectron distribution for varying delay times and subsequently 

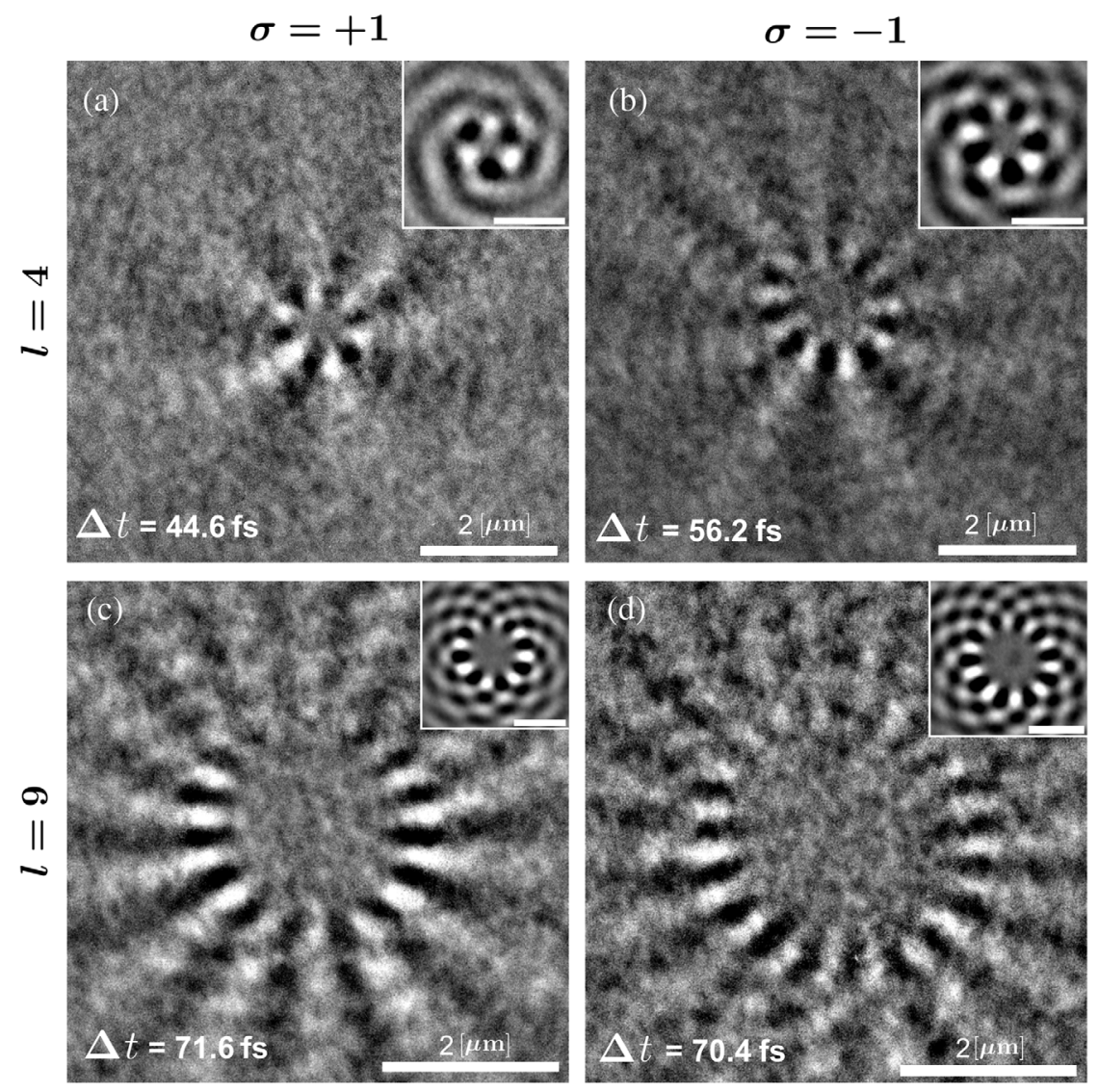

FIG. 4. Experimental results of second-order photon-plasmon AM mixing. We show the filtered raw experimental data for oscillations of $2 \omega \Delta t$. The results clearly show azimuthal patterns of order $l_{\mathrm{el}}=2(l-\sigma)$. The figures are arranged as in Fig. 3 . The insets show the same raw data filtered for oscillations of $\omega \Delta t$ with azimuthal patterns of order $l_{\mathrm{el}}=l-\sigma$. The inset scale bar is 2 microns.

perform a pixel-wise band-pass filtering about the constituent frequencies $\omega$ and $2 \omega$ [Fig. 1(d)]. We apply this delay-time frequency decomposition approach to the sequences of raw signals resulting from plasmonic vortices of order $l=4$ and $l=9$ interacting with circularly polarized light fields (snapshots in Fig. 3). The results in Fig. 4 clearly show the electronic azimuthal patterns, indicating the subtraction (insets) and twice the subtraction of the plasmonic OAM and light SAM, respectively, corresponding to the two types of involved interactions (see SM [40] for full movies).

The filtering process described above is also instrumental for removing the background terms, which may impede the visibility of the clean electron distributions due to the angular-momentum mixing. This case is demonstrated by analyzing two interaction scenarios of plasmon vortices and light pulses with $l=2, \sigma=-1$ and $l=4, \sigma=+1$, respectively, that should yield the same mixing outcome, according to Eq. (4), but exhibit different patterns in the raw photoemission signal [snapshots in Figs. 5(a) and 5(e)]. The spectrum (blue line) of the $\Delta t$ delay-time sequence values of a sample pixel has three distinct peaks. Filtering the raw data about these peaks decomposes the signal into the constituent terms. The spatial distribution of the zero-frequency term [Figs. 5(b) and 5(f)] is contributed mainly by distinct two-photon and two-plasmon absorption events. As the light field is spatially uniform, the distribution is determined by the plasmonic vortices and is thus different for vortices of order 2 and 4 [Fig. 5(b) vs Fig. 5(f)], resembling a scanning near-field microscopy measurement of the vortex field. The first-order lightplasmon mixing is resolved around the $\omega$-centered peak [Figs. 5(c) and 5(g)], showing the $l_{\mathrm{el}}=l-\sigma=3$ azimuthal distribution that is identical for both scenarios. Finally, the second-order light-plasmon mixing is resolved by filtering the $2 \omega$-centered peak [Figs. 5(d) and 5(h)]. It shows an $l_{\mathrm{el}}=2(l-\sigma)=6$ lobed azimuthal distribution identical for both scenarios, unveiling the second-order plasmon-photon mixing. (For full movies, see the SM [40]). The retrieval of complex spatial distributions by pixel-wise filtering emphasizes the fact that we measure local light-matter interactions that adhere to fundamental optical orbital angular-momentum selection rules.

Finally, the electronic angular distributions provide us with handles to discriminate some of the intrinsic electronic interaction paths. We describe the dynamics of two-photon absorption by a three-level model system and its corresponding density matrix [Fig. 5(I)]. The transition from the 

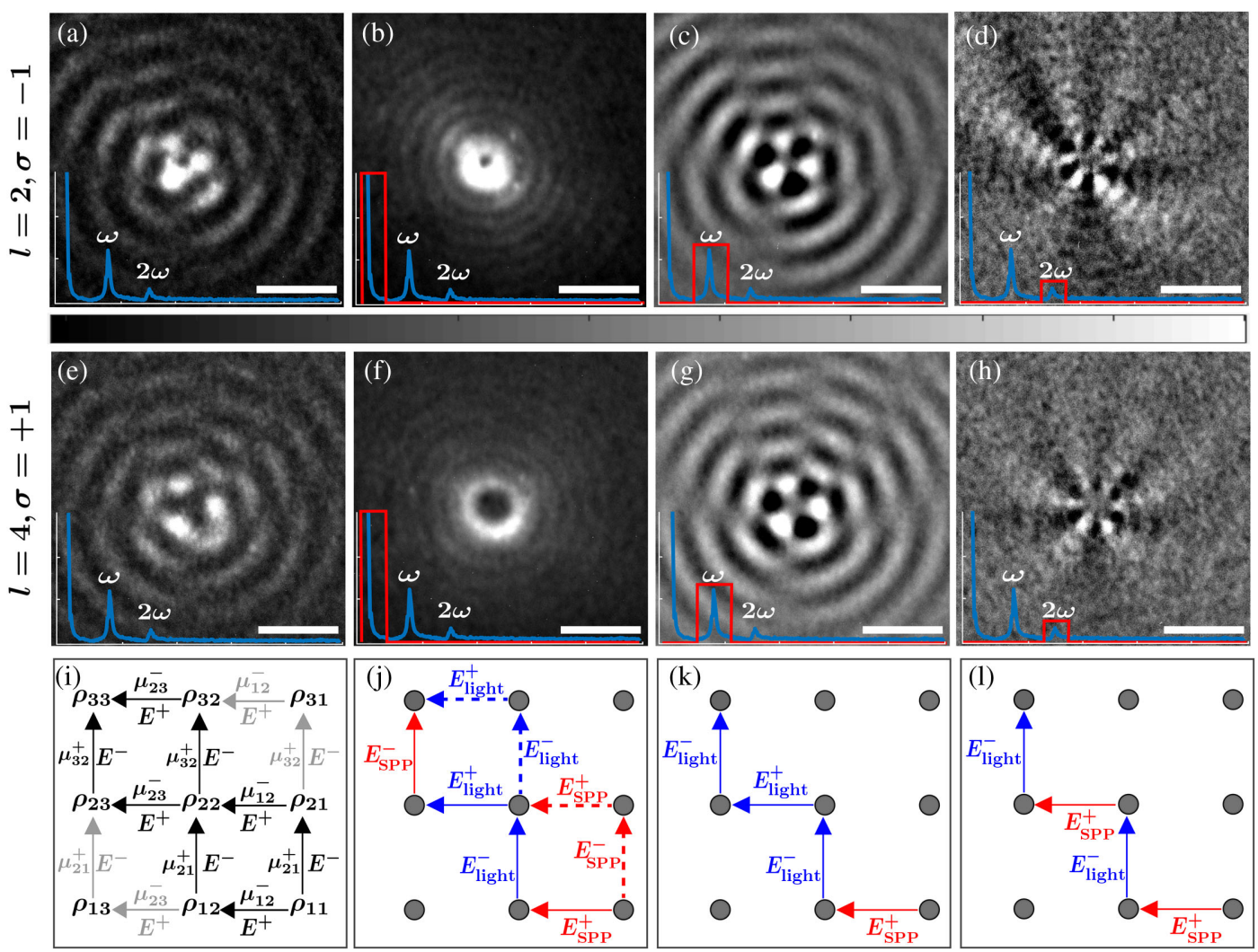

FIG. 5. Delay-time frequency decomposition of the experimental data. Demonstration of two interaction scenarios: $l=2, \sigma=-1$ [(a)—(d)] and $l=4, \sigma=+1[(\mathrm{e})-(\mathrm{h})]$. The raw experimental data at $\Delta t=63.2 \mathrm{fs}$ (a,e) shows three azimuthal lobes with different radial distributions. The spectrum of a sample pixel $\Delta t$ data (blue trace) shows three distinct peaks. Filtering the data about these peaks (red schematic) results in the zero-frequency terms $(\mathrm{b}, \mathrm{f})$, as well as the dynamic first-order (c,g) and second-order (d,h) lightplasmon angular-momentum mixing terms. The scale bar is 2 microns. For full movies, see the SM [40]. (i) - (1) Visualization of the possible quantum pathways in a three-level density matrix corresponding to the decomposed data components. (i) The four-step twophoton absorption process occurring either through the intermediate-state population (black arrows) or via off-diagonal elements (greyed arrows). Example excitation pathways of (j) nonmixing (dashed arrows) and nonoscillating double mixing, (k) single mixing, and (l) double mixing, corresponding to $(b, f),(c, g)$, and $(d, h)$, respectively.

ground to the third excited state may occur through a population of an intermediate state, or through coherences only, without passing through the diagonal elements. Because of the rapid dephasing of the electronic states in metals [48], the quantum pathways corresponding to the latter [greyed arrows in Fig. 5(I)] are suppressed and do not contribute significantly to the measured signal. All the remaining quantum pathways are comprised of the following four steps: generation of off-diagonal coherence between the initial and the intermediate level, population of the intermediate level, generation of coherence between the intermediate and final states, and the eventual population of the final states. Any of these four steps can be driven by $E_{\mathrm{SPP}}^{+}, E_{\mathrm{SPP}}^{-}, E_{\text {light }}^{+}$, and $E_{\text {light }}^{-}$, conforming with the black pathways of Fig. 5(i). Four types of pathways can be resolved: The first type of pathways are those, in which no mixing events take place, where both absorptions occur due to pure photon or plasmon fields [e.g., Fig. 5(j), dashed arrows]. The second type consists of a single angular-momentum mixing in one absorption, with the two steps of the other absorption performed by pure photon or plasmon fields. These are related to the first term of Eq. (4) and exemplified in Fig. 5(k) (the third type) when both transitions are performed by identical two-step sequence of mixing plasmon and light. They are related to the second term of Eq. (4) and exemplified in Fig. 5(1). Finally, the fourth pathway is again a double-mixing event, where the two absorptions are performed by the mutually inverse, plasmon-light, two-step sequence. These absorptions are related to the last term of Eq. (4) and exemplified by solid arrows in Fig. 5(j), where the first coherence is generated by the plasmon and the population by light while the second coherence is generated by light and the final-state population by the plasmon. The latter process, however, cannot be easily resolved experimentally. It has no dependence on time delay $\Delta t$ and does not exhibit any azimuthal dependence; it is overlaid with the rest of the static background terms, and because it has a lower amplitude, it is covered by the 
more dominant spatial distribution of the out-of-plane field component of the plasmonic vortex field [see Appendix C and Figs. 5(b) and 5(f)].

\section{CONCLUSIONS}

To summarize, we presented experimental observations and theoretical analysis of spin-orbit mixing between two fields carrying two different types of angular momenta. Using photoionization as the mixing process, we demonstrated a subtractive spin-orbit mixing effect. We showed that the effect fundamentally stems from the absorptive nature of the mixing mechanism by mixing between the positive and negative frequencies of the field. Moreover, using pixel-wise time-delay-frequency decomposition, we experimentally extracted a second-order plasmon-photon mixing effect consisting of two mixing events. The two mixing processes allow us to experimentally classify and study the quantum pathways undertaken by the emitted electrons. Our results thus present light-matter interaction effects that adhere to fundamental selection rules of optical orbital angular momentum. Our findings provide understanding of angular-momenta interactions and may have an impact on advanced light-matter interactions, electronic sources, and coherent control. Furthermore, using tight high-order plasmonic vortices to potentially deliver the high angular momentum required for ultrafast demagnetization can become a great advantage for heat-assisted magnetic recording.

\section{ACKNOWLEDGMENTS}

G. S. and M. O. acknowledge support from the Israeli Centers of Research Excellence "Circle of Light." We acknowledge the Micro-Nano Fabrication Unit (MNFU) Technion for support with sample fabrication. D. K. acknowledges funding from the Irish Research Council and the Marie Curie Actions ELEVATE fellowship. A. K. M., M.H., E. P., and M. A. acknowledge funding from the DFG within the program SFB/TRR 173: "SPIN $+\mathrm{X}$." We acknowledge the Nano Structuring Center Kaiserslautern for support with the sample fabrication. E. P. acknowledges funding from the DFG through the Excellence Initiative by the Graduate School Materials Science in Mainz (GSC 266).

\section{APPENDIX A: MATERIALS AND METHODS}

\section{Sample fabrication}

The polycrystalline gold samples were prepared by sputtering a 200-nm-thick gold layer onto a silicon substrate serving as a conducting substrate to avoid accumulation of electrostatic charges on the sample during the PEEM measurements. The slits in the gold films for generating the vortex were milled with FIB using Ga ions (FEI Helios Nanolab 600) through the gold layer.

\section{Time-resolved two-photon photoemission electron microscopy}

The PEEM system in our experiment is the FOCUS GmbH IS-PEEM, with about 30-nm spatial resolution. The microscope uses an electrostatic lens system to map the lateral variations in the photoemission yield from the nanostructured surface. The light source is a Ti:sapphire oscillator (Spectra Physics Tsunami), which produces pulses with a duration of about $23 \mathrm{fs}$ at a central wavelength of $800 \mathrm{~nm}$. The pump and probe pulses were produced using an actively phase-stabilized Mach-Zehnder interferometer based on the Pancharatnam phase [49] with a time step, $\Delta t$ resolution of 30 attoseconds [41]. The light pulses are launched at a normal incidence onto the gold sample; the pump pulse launches the plasmonic vortex, and after a time $\Delta t$, the probe pulse interacts with the alreadypropagating vortex, liberating the photoelectrons, which are then collected by the electron optics. The spot diameter of the pump and probe beams on the sample is about $100 \mu \mathrm{m}$.

\section{Plasmon-vortex excitation}

Plasmonic vortices are formed in our experiments by a short, circularly polarized pulse $(<23 \mathrm{fs})$ with $800-\mathrm{nm}$ central wavelength, normally impinging on a plasmonic vortex generator [7] (PVG) of order $m$. The PVG structure consists of $m$ segments of a spiral of the form $r_{m}(\theta)=$ $r_{i}+\lambda_{\mathrm{SPP}} \cdot \bmod (m \theta, 2 \pi) / 2 \pi$ engraved on a gold surface, where $\lambda_{\text {SPP }}$ is the plasmonic wavelength, $\theta$ is the azimuthal coordinate and $r_{i}$ is the inner radius. In our experiments on the gold-vacuum surface $\lambda_{\mathrm{SPP}} \sim 784 \mathrm{~nm}$, the inner radii of the structures are $r_{i}=17 \mu \mathrm{m}$ for the PVG of $m=3$ and $r_{i}=18 \mu \mathrm{m}$ for the PVG of $m=10$. The width of the engraved slits is $400 \mathrm{~nm}$. Figure 2 depicts the simulated snapshots of the spatial distributions of the plasmonic vortex field components initiated within a PVG of order $m=3$ and $m=10$. We see the formation of a vortex of order $l=4$ and $l=9$, respectively. The angularmomentum index of the resulting vortex, $l$, is the combinaItion of the order of the spiral $m$ and the spin of the generating light field $\sigma_{\text {init }}=+/-1$, resulting in $l=m+\sigma_{\text {init }}$. PVG of order $m=10$ was also used in Ref. [10].

It is important to note that this linear conversion process occurs upon the interaction of the illumination with the structure boundaries. Once the SPPs are launched with the proper phases by the boundary, the topological charge of the to-be-formed vortex is predetermined.

\section{APPENDIX B: SPP EXCITATION EFFICIENCY}

The efficiency of plasmonic coupling is determined by the geometry of the coupling slit and has been investigated. It has been shown theoretically [50], for example, that an approximately $45 \%$ efficiency could be achieved in an optimized freestanding slit case. It should be noted, 
however, that in our paper, a thorough maximization of the coupled signal through the optimization of the slit form was not a prime goal.

We numerically modeled our configuration as a rectangular slit milled in a 200-nm-thick gold layer (Johnson and Christy) on a silicon substrate (Palik). Based on our detailed finite-difference time-domain calculations, the percentage of light to plasmon intensity coupling (for our configuration) is $22 \%$ (intensity), and it is located near the peak coupling power for this geometry (see Fig. 6).

The coupling power is calculated as follows: In order to avoid erroneously high coupling results due to the transient response of the slit in the vicinity of the slit [51], the power is collected 25.1 microns away from the boundary of the slit over a $875-\mathrm{nm}$ cross section, $800 \mathrm{~nm}$ from the gold surface into the air and $75 \mathrm{~nm}$ into the gold. Finally, to account for the propagation losses of the SPPs, the resulting power is normalized by the propagation decay factor calculated from the simulated gold parameters: $\exp \left[-2 \operatorname{Im}\left(k_{\mathrm{SPP}}\right)\right.$. $\left.25.1 \times 10^{-6}\right] \approx 0.58$. The efficiency is then calculated by normalizing the collected fractional source power by the fill factor of the slit with respect to the source.

We should note that, to avoid scattered field penetration, we kept the collection monitor to $800 \mathrm{~nm}$, which further slightly reduces the actual coupled power. Furthermore, we should note that the model neglects geometric deviations of

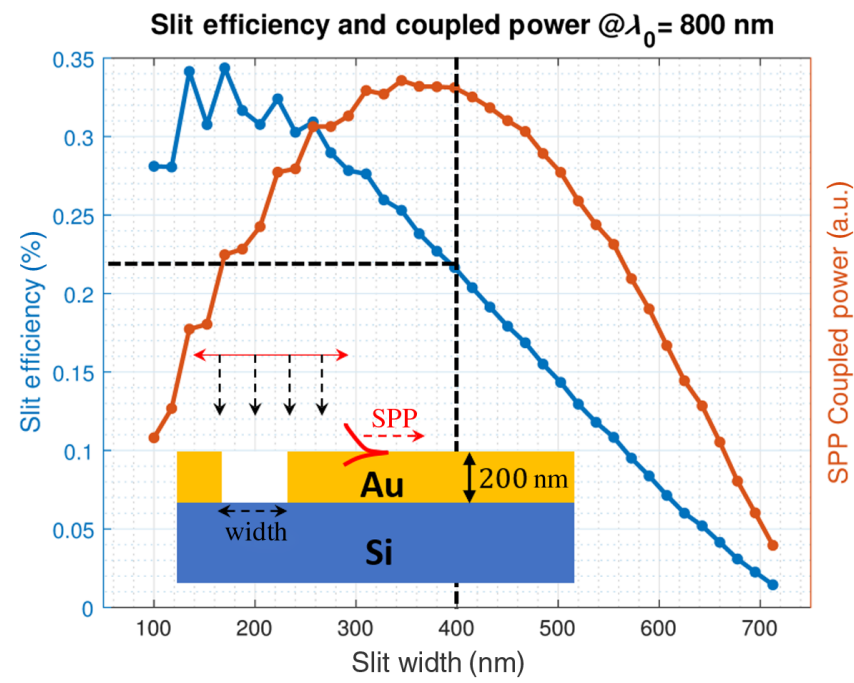

FIG. 6. Modeling of the coupling efficiency of a rectangular slit milled in gold on a silicon substrate under illumination by a horizontally polarized plane wave at normal incidence (red arrow for polarization and dashed black for propagation direction). The red schematic in the inset is the SPP field profile propagating on the gold-air interface. The blue curve represents the intrinsic slit coupling efficiency with the coupled power normalized by the area (width) of the slit relative to the area of the source (fill factor). The orange curve represents the power coupled to the SPPs in arbitrary units. The dashed black curves mark our condition slit width of $400 \mathrm{~nm}$, resulting in an efficiency of $22 \%$ and roughly maximal coupling power. the slit from perfect rectangular form, which, in turn, should probably deteriorate the actual slit performance. In addition, we neglect here the native $\mathrm{SiO}_{2}$ layer on the silicon-gold interface, which is typically about $2 \mathrm{~nm}$ thick. The noisy values for narrow slits originate from numerical errors due to the finite mesh. Finally, in our experiment, we did not set a goal to maximize the coupling efficiency of the slit (even though we are seemingly near the maximum for this configuration).

We attribute this quite high coupling efficiency to the Fabry-Perot-like behavior of the slit-guided mode in the direction parallel to the surface normal and the effectively high reflectivity of the air-silicon interface in the bottom part of the slit. By optimizing the thickness of the golden layer, we can potentially further increase the coupling efficiency. But this was not our goal here.

Notably, the $22 \%$ is the power efficiency, and the field coefficient is therefore $\sqrt{0.22}=0.47$.

\section{APPENDIX C: VECTOR-FIELD-COMPONENTS CONTRIBUTIONS}

As seen in Figs. 5(a) and 5(e), the signals obtained from the two distinct mixing scenarios both have three azimuthal lobes; however, their radial distribution is clearly different. While the number of lobes is precisely described by the first term of Eq. (4), the radial distribution deviates from it.

To explain this disparity, we must consider the effect and origin of the background terms. The dominant background term (see SM [40]) is $\left(\left|\vec{E}_{\text {light }}\right|^{2}+\left|\vec{E}_{\mathrm{SPP}}(\vec{r})\right|^{2}\right)^{2}$. The light is spatially uniform, and thus, the plasmonic term dominates the time-delay-independent spatial distribution of the background. Moreover, we show that it is the out-of-plane field component $\left(E_{z}\right)$ that dominates this distribution.

To prove this assertion, we divide the PEEM signal into three time-delay regimes, defined by the pulse duration and characteristic dimensions of the geometry studied, as discussed in Ref. [10]. We define the early [Fig. 7(a)] pattern before the formation of the vortex in the center, the evolved [Fig. 7(b)] pattern when the vortex is in the revolution stage, and the late pattern [Fig. 7(c)] when the vortex has already dissolved. We see that, already for
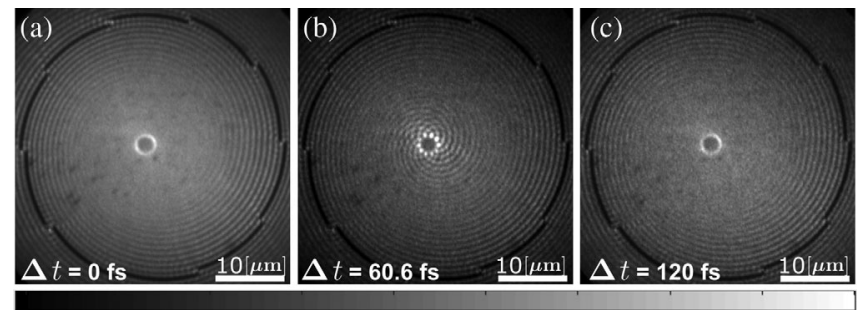

FIG. 7. Measured PEEM snapshots of an $l=9$ vortex interacting with a $\sigma=-1$ light field. The snapshots show time delays of (a) $\Delta t=0 \mathrm{fs}$ (early), (b) $\Delta t=60.6 \mathrm{fs}$ (revolving) and (c) $\Delta t=120$ fs (late). 
zero plasmon-light launching delay [Fig. 7(a)], there is a bright circle appearing in the center [10]. Such a bright, static circle also appears in the late pattern.

We then conducted a fitting procedure (see SM [40]) to obtain the radius of the circular field distribution of the pattern in the center of the structure as a function of $\Delta t$. The presented measurements were done on vortex generators of order 10 in order to obtain high-order vortices (with $l=11$ or $l=9$ depending on the excitation [Fig. 8]) having more samples over their perimeter, resulting in higher fit accuracy.

We clearly see that the late static patterns fall to within $0.5 \%$ on the radius corresponding to the out-of-plane $\left(E_{z}\right)$ component of the interacting vortex field. Thus, we confirm that the origin of the bright central photoemission circle is from the plasmon-plasmon photoemission, having a spatial distribution of $J_{l}\left(k_{\mathrm{SPP}} r\right)$, with $l$ being the order of the SPP vortex. We also observe that, in the early evolution pattern, the radial distribution oscillates around a mean that corresponds to the out-of-plane pattern (late static pattern), for about $37 \mathrm{fs}$ after $\Delta t=0$; in the dynamic region, we can see that the radial distribution is "pulled" towards the radial distribution of the mixing term $\propto \vec{E}_{\mathrm{SPP}} \cdot \vec{E}_{\sigma^{ \pm}}$, which is governed by the in-plane components, with a radial distribution of $J_{l \mp 1}\left(k_{\mathrm{SPP}} r\right)$.

The early region shows oscillations of the radial pattern that are attributed to the cross-correlation between the stilloverlapping tails of the plasmon and light pulses. Finally, for large delays where the SPPs and light pulses do not temporally overlap, the oscillations disappear to reveal only

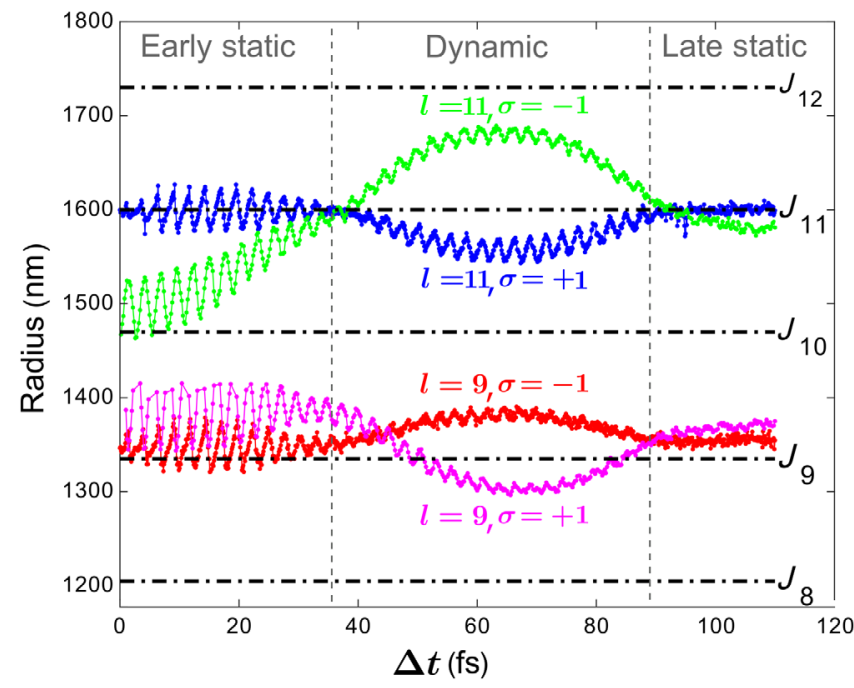

FIG. 8. Radius of the central pattern as a function of the delay, $\Delta t$. The horizontal dashed lines represent the radii of the main lobes of the corresponding Bessel functions $\left[J_{8}\left(k_{\mathrm{SPP}} r\right)-J_{12}\left(k_{\mathrm{SPP}} r\right)\right]$. Each curve corresponds to a vortex-light configuration presented in Table I. The experimental data for the curves are presented in the insets of Figs. 3(c) and 3(d) in the main text and in Figs. S2 C and S2 D of SM [40]. The blue curve is obtained by creating a vortexlight configuration that was also used to observe the dynamics of a plasmonic vortex in Ref. [10].
TABLE I. A summary of radial distributions describing the results in Fig. 8. The table is arranged according to the line colors of the data, showing the vortex order $l_{\mathrm{SPP}}$ and the light spin $\sigma_{\text {light }}$ participating in the interaction, and the predicted radial dependence of the out-of-plane vortex field component $\left(E_{z}\right)$ and the corresponding mixing term. The rightmost column points to the figures with the experimental data snapshots.

\begin{tabular}{lcccc}
\hline \hline Line & $l_{\mathrm{SPP}}$, & $\begin{array}{c}E_{z} \text { radial } \\
\text { distribution } \\
\text { color }\end{array}$ & $\begin{array}{c}\text { Mixing radial } \\
\text { distribution }\end{array}$ & \\
\hline Magenta & $9,+1$ & $J_{l_{\mathrm{SPP}}}\left(k_{\mathrm{SPP}} r\right)$ & $J_{l-\sigma}\left(k_{\mathrm{SPP}} r\right)$ & Figure \\
Red & $9,-1$ & $J_{9}\left(k_{\mathrm{SPP}} r\right)$ & $J_{8}\left(k_{\mathrm{SPP}} r\right)$ & $3(\mathrm{c})$ \\
Blue & $11,+1$ & $J_{11}\left(k_{\mathrm{SPP}} r\right)$ & $J_{10}\left(k_{\mathrm{SPP}} r\right)$ & $3(\mathrm{~d})$ \\
Green & $11,-1$ & $J_{11}\left(k_{\mathrm{SPP}} r\right)$ & $J_{12}\left(k_{\mathrm{SPP}} r\right)$ & $\mathrm{S} 2 \mathrm{C}$ \\
\hline \hline
\end{tabular}

the late signature of the out-of-plane component photoemission originating from plasmon-only photoemission [47].

Overall, we can see that the radial distribution in the raw signal is altered by the background term corresponding mainly to the out-of-plane field component. Finally, we note that, probably due to a drift in one of our experiments, the green curve does not oscillate around a constant mean in the early pattern.

We filter the signal as described above and in the main text around $\omega$ delay-time oscillations and fit the central radial distribution of the filtered signal (Fig. 9). The radial distributions of the filtered signal become decoupled from the static background and are now flattened and oscillate around the proper Bessel function radii throughout the whole dynamic region as predicted by Eq. (4).

Thus, both the radial and azimuthal distributions are well described by Eq. (4).

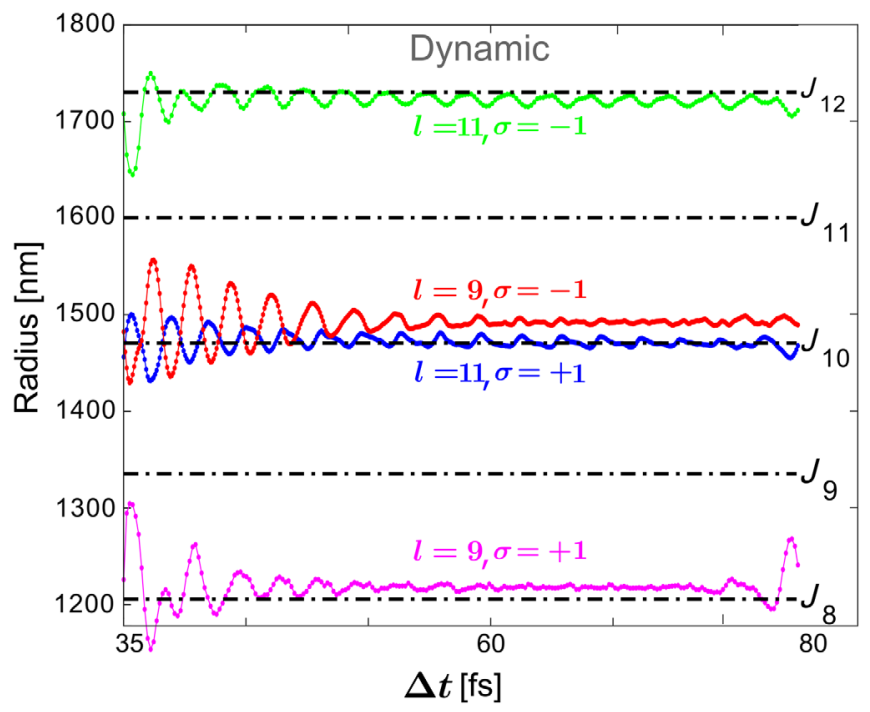

FIG. 9. Radius of the $\omega \Delta t$ filtered central pattern in the dynamic region. The horizontal dashed lines represent the radii of the main lobes of the corresponding Bessel functions as in Fig. 8. 
[1] L. Allen, M. W. Beijersbergen, R. Spreeuw, and J. Woerdman, Orbital Angular Momentum of Light and the Transformation of Laguerre-Gaussian Laser Modes, Phys. Rev. A 45, 8185 (1992).

[2] A. M. Yao and M. J. Padgett, Orbital Angular Momentum: Origins, Behavior and Applications, Adv. Optics Photonics 3, 161 (2011).

[3] D. L. Andrews and M. Babiker, The Angular Momentum of Light (Cambridge University Press, Cambridge, England, 2012), https://doi.org/10.1017/CBO9780511795213.

[4] K. Bliokh, F. J. Rodriguez-Fortuno, F. Nori, and A. V. Zayats, Spin-Orbit Interaction of Light, Nat. Photonics 9, 796 (2015).

[5] F. Cardano and L. Marrucci, Spin-Orbit Photonics, Nat. Photonics 9, 776 (2015).

[6] Y. Gorodetski, A. Niv, V. Kleiner, and E. Hasman, Observation of the Spin-Based Plasmonic Effect in Nanoscale Structures, Phys. Rev. Lett. 101, 043903 (2008).

[7] H. Kim, J. Park, S.-W. Cho, S.-Y. Lee, M. Kang, and B. Lee, Synthesis and Dynamic Switching of Surface Plasmon Vortices with Plasmonic Vortex Lens, Nano Lett. 10, 529 (2010).

[8] C.-F. Chen, C.-T. Ku, Y.-H. Tai, P.-K. Wei, H.-N. Lin, and C.-B. Huang, Creating Optical Near-Field Orbital Angular Momentum in a Gold Metasurface, Nano Lett. 15, 2746 (2015).

[9] A. David, B. Gjonaj, and G. Bartal, Two-Dimensional Optical Nanovortices at Visible Light, Phys. Rev. B 93, 121302 (2016).

[10] G. Spektor et al., Revealing the Subfemtosecond Dynamics of Orbital Angular Momentum in Nanoplasmonic Vortices, Science 355, 1187 (2017).

[11] G. Rosenblatt, E. Feigenbaum, and M. Orenstein, Circular Motion of Electromagnetic Power Shaping the Dispersion of Surface Plasmon Polaritons, Opt. Express 18, 25861 (2010).

[12] K. Y. Bliokh, A. Y. Bekshaev, and F. Nori, Extraordinary Momentum and Spin in Evanescent Waves, Nat. Commun. 5, 3300 (2014).

[13] Y. Gorodetski, N. Shitrit, I. Bretner, V. Kleiner, and E. Hasman, Observation of Optical Spin Symmetry Breaking in Nanoapertures, Nano Lett. 9, 3016 (2009).

[14] G. Spektor, A. David, G. Bartal, M. Orenstein, and A. Hayat, Spin-Patterned Plasmonics: Towards Optical Access to Topological-Insulator Surface States, Opt. Express 23, 32759 (2015).

[15] A. De Hoogh, N. Rotenberg, and L. Kuipers, Optical Singularities in Plasmonic Fields Near Single Subwavelength Holes, J. Optics 16, 114004 (2014).

[16] W.-Y. Tsai, J.-S. Huang, and C.-B. Huang, Selective Trapping or Rotation of Isotropic Dielectric Microparticles by Optical Near Field in a Plasmonic Archimedes Spiral, Nano Lett. 14, 547 (2014).

[17] M. L. Juan, M. Righini, and R. Quidant, Plasmon Nanooptical Tweezers, Nat. Photonics 5, 349 (2011).

[18] R. Quidant and C. Girard, Surface-Plasmon-Based Optical Manipulation, Laser Photonics Rev. 2, 47 (2008).

[19] N. Rivera, I. Kaminer, B. Zhen, J. D. Joannopoulos, and M. Soljačić, Shrinking Light to Allow Forbidden Transitions on the Atomic Scale, Science 353, 263 (2016).
[20] A. Picón, A. Benseny, J. Mompart, J. R. V. de Aldana, L. Plaja, G. F. Calvo, and L. Roso, Transferring Orbital and Spin Angular Momenta of Light to Atoms, New J. Phys. 12, 083053 (2010).

[21] N. Shitrit, I. Bretner, Y. Gorodetski, V. Kleiner, and E. Hasman, Optical Spin Hall Effects in Plasmonic Chains, Nano Lett. 11, 2038 (2011).

[22] G. Spektor, A. David, B. Gjonaj, G. Bartal, and M. Orenstein, Meta Focusing by Meta-Spiral Plasmonic Lens, Nano Lett. 15, 5739 (2015).

[23] J. Lin, J. P. B. Mueller, Q. Wang, G. Yuan, N. Antoniou, X.-C. Yuan, and F. Capasso, Polarization-Controlled Tunable Directional Coupling of Surface Plasmon Polaritons, Science 340, 331 (2013).

[24] F. J. Rodriguez-Fortuño, G. Marino, P. Ginzburg, D. O'Connor, A. Martinez, G. A. Wurtz, and A. V. Zayats, Near-Field Interference for the Unidirectional Excitation of Electromagnetic Guided Modes, Science 340, 328 (2013).

[25] D. O'Connor, P. Ginzburg, F. Rodriguez-Fortuño, G. Wurtz, and A. Zayats, Spin-Orbit Coupling in Surface Plasmon Scattering by Nanostructures, Nat. Commun. 5, 5327 (2014).

[26] K. Dholakia, N. Simpson, M. Padgett, and L. Allen, SecondHarmonic Generation and the Orbital Angular Momentum of Light, Phys. Rev. A 54, R3742 (1996).

[27] L. J. Pereira, W. T. Buono, D. S. Tasca, K. Dechoum, and A. Z. Khoury, Orbital Angular Momentum Mixing in Type II Second Harmonic Generation, in Nonlinear Optics (Optical Society of America, 2017), paper NM3B.2, https://doi.org/ 10.1364/NLO.2017.NM3B.2.

[28] J. Courtial, K. Dholakia, L. Allen, and M. Padgett, SecondHarmonic Generation and the Conservation of Orbital Angular Momentum with High-Order Laguerre-Gaussian Modes, Phys. Rev. A 56, 4193 (1997).

[29] G. Gariepy, J. Leach, K. T. Kim, T. J. Hammond, E. Frumker, R. W. Boyd, and P. B. Corkum, Creating HighHarmonic Beams with Controlled Orbital Angular Momentum, Phys. Rev. Lett. 113, 153901 (2014).

[30] X. Y. Xiong, A. Al-Jarro, L. J. Jiang, N. C. Panoiu, and E. Wei, Mixing of Spin and Orbital Angular Momenta via Second-Harmonic Generation in Plasmonic and Dielectric Chiral Nanostructures, Phys. Rev. B 95, 165432 (2017).

[31] D. P. Caetano, M. P. Almeida, P. H. S. Ribeiro, J. A. O. Huguenin, B. C. dos Santos, and A. Z. Khoury, Conservation of Orbital Angular Momentum in Stimulated DownConversion, Phys. Rev. A 66, 041801 (2002).

[32] G. X. Li, S. M. Chen, Y. Cai, S. Zhang, and K. W. Cheah, Third Harmonic Generation of Optical Vortices Using Holography-Based Gold-Fork Microstructure, Adv. Opt. Mater. 2, 389 (2014).

[33] N. V. Bloch, K. Shemer, A. Shapira, R. Shiloh, I. Juwiler, and A. Arie, Twisting Light by Nonlinear Photonic Crystals, Phys. Rev. Lett. 108, 233902 (2012).

[34] G. Li, S. Zhang, and T. Zentgraf, Nonlinear Photonic Metasurfaces, Nat. Rev. Mater. 2, 17010 (2017).

[35] M. Tymchenko, J. S. Gomez-Diaz, J. Lee, N. Nookala, M. A. Belkin, and A. Alù, Gradient Nonlinear PancharatnamBerry Metasurfaces, Phys. Rev. Lett. 115, 207403 (2015). 
[36] S. Keren-Zur, O. Avayu, L. Michaeli, and T. Ellenbogen, Nonlinear Beam Shaping with Plasmonic Metasurfaces, ACS Photonics 3, 117 (2016).

[37] A. E. Minovich, A. E. Miroshnichenko, A. Y. Bykov, T. V. Murzina, D. N. Neshev, and Y. S. Kivshar, Functional and Nonlinear Optical Metasurfaces, Laser Photonics Rev. 9, 195 (2015).

[38] H. Raether, Surface Plasmons on Smooth Surfaces, in Surface Plasmons on Smooth and Rough Surfaces and on Gratings, Springer Tracts in Modern Physics Vol. 111 (Springer, Berlin, Heidelberg, 1988), pp. 4-39.

[39] S. A. Maier, Plasmonics: Fundamentals and Applications (Springer, New York, 2007), https://www.springer.com/gp/ book/9780387331508.

[40] See Supplemental Material at http://link.aps.org/ supplemental/10.1103/PhysRevX.9.021031 for more detailed analytic derivation and numeric modeling, experimental as well as simulation movies with accompanying description, additional experimental data and detailed description of radial fitting and delay-time frequency decomposition procedures.

[41] M. Wehner, M. Ulm, and M. Wegener, Scanning Interferometer Stabilized by Use of Pancharatnam's Phase, Opt. Lett. 22, 1455 (1997).

[42] M. Bauer, C. Wiemann, J. Lange, D. Bayer, M. Rohmer, and M. Aeschlimann, Phase Propagation of Localized Surface Plasmons Probed by Time-Resolved Photoemission Electron Microscopy, Appl. Phys. A 88, 473 (2007).

[43] A. Kubo, N. Pontius, and H. Petek, Femtosecond Microscopy of Surface Plasmon Polariton Wave Packet
Evolution at the Silver/Vacuum Interface, Nano Lett. 7, 470 (2007).

[44] M. I. Stockman, M. F. Kling, U. Kleineberg, and F. Krausz, Attosecond Nanoplasmonic-Field Microscope, Nat. Photonics 1, 539 (2007).

[45] P. Kahl et al., Direct Observation of Surface Plasmon Polariton Propagation and Interference by Time-Resolved Imaging in Normal-Incidence Two Photon Photoemission Microscopy, Plasmonics 13, 239 (2017).

[46] B. Frank et al., Short-Range Surface Plasmonics: Localized Electron Emission Dynamics from a 60- $\mathrm{nm}$ Spot on an Atomically Flat Single-Crystalline Gold Surface, Sci. Adv. 3, e1700721 (2017).

[47] D. Podbiel, P. Kahl, and F.-J. M. zu Heringdorf, Analysis of the Contrast in Normal-Incidence Surface Plasmon Photoemission Microscopy in a Pump-Probe Experiment with Adjustable Polarization, Appl. Phys. B 122, 90 (2016).

[48] M. J. Weida, S. Ogawa, H. Nagano, and H. Petek, Ultrafast Interferometric Pump-Probe Correlation Measurements in Systems with Broadened Bands or Continua, J. Opt. Soc. Am. B 17, 1443 (2000).

[49] S. Pancharatnam, Generalized Theory of Interference, and Its Applications. Part I. Coherent Pencils, Proc. Indian Acad. Sci. A 44, 247 (1956).

[50] P. Lalanne, J. Hugonin, and J. Rodier, Approximate Model for Surface-Plasmon Generation at Slit Apertures, JOSA A 23, 1608 (2006).

[51] Y. Gravel and S. Yunlong, Rigorous solution for the transient surface plasmon polariton launched by subwavelength slit scattering, Opt. Express 16, 21903 (2008). 